\title{
The importance of the aggregation of ticks on small mammal hosts for the establishment and persistence of tick-borne pathogens: an investigation using the $R_{0}$ model
}

\author{
ALAN HARRISON ${ }^{1}$ * and NIGEL C. BENNETT ${ }^{1,2}$ \\ ${ }^{1}$ Department of Zoology and Entomology, University of Pretoria, Pretoria, South Africa \\ ${ }^{2}$ Department of Zoology, King Saud University, Riyadh, Kingdom of Saudi Arabia
}

(Received 26 Fanuary 2012; revised 30 March 2012; accepted 27 April 2012)

\begin{abstract}
SUMMARY
Aggregation of parasites amongst hosts is important for the epidemiology of vector-borne diseases because hosts that support the majority of the vector population are responsible for the majority of pathogen transmission. Ixodes ricinus ticks transmit numerous pathogens of medical importance including Borrelia burgdorferi s.l. and tick-borne encephalitis virus. One transmission route involved is 'co-feeding transmission', where larvae become infected via feeding alongside infected nymphs. The aggregation of ticks on hosts leads to an increase in the number of larvae feeding alongside nymphs, increasing the transmission potential via this route. The basic reproduction number, $R_{0}$, can be used to identify whether a pathogen will become established if introduced. In the current study we use previously published tick, and pathogen, specific data to parameterize an $R_{0}$ model to investigate how the degree of aggregation of ticks on hosts affects pathogen persistence. The coincident aggregated distribution permitted the establishment of tick-borne encephalitis virus but did not influence whether B. burgdorferi s.l. became established. The relationship between the $k$-exponent of the negative binomial distribution and $R_{0}$ was also defined. Therefore, the degree of aggregation of ticks on small mammal hosts has important implications for the risk to human health in a given area.
\end{abstract}

Key words: vector-borne diseases, aggregation, transmission, Ixodes ricinus, Borrelia burgdorferi s.l., epidemiology.

\section{INTRODUCTION}

Parasites are often aggregated among their hosts such that a large proportion of the parasite population is present on only a small proportion of the host population (Crofton, 1971; Dobson and Merenlender, 1991; Shaw and Dobson, 1995). This type of aggregation follows a statistical trend found across a wide range of disciplines known as the 'Pareto principle' or ' $80 / 20$ rule' $(80 \%$ of parasites are found on $20 \%$ of the host population) (Woolhouse et al. 1997; Koch, 1999). The aggregated distribution of parasites can often be modelled using the negative binomial distribution, a discrete probability distribution with a $k$-exponent that defines the degree of aggregation (Crofton, 1971; Shaw and Dobson, 1995). Heterogeneities in thse abundance of parasites among hosts arise due to variability in individuals in their rate of exposure to parasites and in their susceptibility to parasites once they have been exposed (Wilson et al. 2002). The aggregation of parasites has important implications for the epidemiology of vector-borne pathogens; if a small fraction of the host population supports the majority of the

* Corresponding author: Department of Zoology and Entomology, University of Pretoria, Private Bag X20, Hatfield, 0028, Pretoria, South Africa. Tel: +27(0) 713815103. E-mail: atharrison@zoology.up.ac.za vector population then those individuals will also be the most important hosts for the transmission of pathogens (Woolhouse et al. 1997; Perkins et al. 2003).

Ticks are vectors of numerous bacterial, viral and protozoan pathogens of medical importance (Jongejan and Uilenberg, 2004) and small mammals are reservoirs of many of these pathogens (Goodman et al. 2005). The transmission of pathogens from one tick to another, a pre-requisite for the establishment of cycles of infection, may occur via 3 pathways (see Randolph (1998) for a review). Firstly, female ticks may transmit the pathogen to eggs via transovarial transmission. Secondly, nymphs may feed on, and infect, a host leading to a systemic infection; larval ticks may then acquire an infection by ingesting the blood of the infected host, maintain the infection transstadially, and infect an alternative host during a subsequent bloodmeal as a nymph. Thirdly, ticks may become infected by co-feeding, spatially or temporally, alongside infected ticks. This 'co-feeding transmission' does not require the host to have a systemic infection but instead pathogens are passed from one tick to another as they feed together. Vertebrate hosts may vary in their competency to support transstadial and co-feeding transmission. For example, red grouse (Lagopus lagopus scoticus) and sheep become viraemic with louping-ill and can 
transmit it transstadially, hares (Lepus timidus), however, do not develop a systemic infection but play a crucial role in the persistence of the disease via non-viraemic, co-feeding transmission (Hudson et al. 1995; Norman et al. 2004). In the case of Borrelia burgdorferi s.l., small mammals are competent for both transstadial transmission and co-feeding transmission. In contrast, sheep do not develop a systemic infection and therefore do not permit transstadial transmission, however, they do support co-feeding transmission (see Randolph et al. (1996) for a review).

Larvae and nymphs of Ixodes ricinus, the vector of numerous pathogens, have been found to be highly aggregated among small mammal hosts across much of Europe, typically having a $k$-exponent of the negative binomial distribution between 0 and 1 (Randolph et al. 1999). Often larvae and nymphs of I. ricinus are not independently aggregated but follow a coincident aggregated distribution, i.e. those individuals with large numbers of larvae also support nymphs (Randolph et al. 1999; Harrison et al. 2011). For example, Harrison et al. (2011) found that the same $20 \%$ of wood mice in Ireland fed $72 \%$ of I. ricinus larvae and all nymphs found on mice, a pattern corresponding closely to the $80 / 20$ rule. This level of aggregation has important implications for the efficiency of co-feeding transmission as it may allow roughly twice as many larvae to feed alongside nymphs than would be possible with an independent aggregated distribution (Randolph et al. 1999).

The basic reproduction number, denoted as $R_{0}$, has a number of important applications in epidemiology. It is defined as the average number of secondary cases caused by one infected individual entering a population consisting solely of susceptible individuals (Anderson and May, 1990; Diekmann et al. 1990). It has a threshold value such that if $R_{0}>1$ a pathogen will persist when introduced, but a value of $R_{0}<1$ suggests that it will die out. In the case of tickborne pathogens it can be used to identify the average number of ticks infected in the second generation after 1 infected tick enters the population, therefore, if $R_{0}>1$, the pathogen will perpetuate within the tickhost system. Heterogeneities in the efficiency of different transmission routes of tick-borne pathogens will affect $R_{0}$ and, therefore, the likelihood of a pathogen becoming established within a system (Woolhouse et al. 1997). As expected, due to the applicability of the basic reproduction number, many attempts have been made to estimate $R_{0}$ for tickborne pathogens (Randolph, 1998; Norman et al. 1999; Randolph et al. 1999; Caraco et al. 2002; Rosa et al. 2003; Ghosh and Pugliese, 2004; Rosa and Pugliese, 2007). Indeed, Rosa et al. (2003) previously investigated the role of tick aggregation on the persistence of tick-borne pathogens; however, many of these approaches were lacking in their relevance to the tick life cycle, assuming bite rates akin to insect vector models, or used derived expressions that could not be strictly interpreted as a biologically meaningful value of $R_{0}$ (Hartemink et al. 2008).

The current study uses the most up-to-date and biologically relevant next-generation model of the basic reproduction number for tick-borne pathogens (Hartemink et al. 2008) to investigate how the degree of aggregation of $I$. ricinus ticks on small mammal hosts (ranging from dispersed to independent aggregated to coincident aggregated distribution) affects the ability of tick-borne pathogens to become established in enzootic small mammal cycles. We use previously published tick-related and pathogenspecific data as well as simulated data to parameterize the $R_{0}$ model specific for Borrelia burgdorferi s.l., and tick-borne encephalitis virus (TBEV), 2 of the most important tick-borne pathogens of humans in Europe. The $k$-exponent of the negative binomial distribution was varied while other parameters were held static so that the effect of the aggregation of ticks on the persistence of tick-borne pathogens in small mammal hosts could be observed. This method also allowed the relationship between the $k$-exponent of the negative binomial distribution and $R_{0}$ to be defined. It has also been shown that increasing the diversity of vertebrate hosts in an ecosystem can reduce the risk of disease as only some hosts are competent for disease transmission and infective tick bites may be 'wasted' on non-transmission competent hosts, the so called 'dilution effect' (Ostfeld and Keesing, 2000; Schmidt and Ostfeld, 2001). Therefore, we also investigated how the degree of aggregation of ticks on small mammal hosts affects the ability of these pathogens to become established in relation to the proportion of transmissioncompetent hosts on which ticks are feeding.

\section{MATERIALS AND METHODS}

\section{Data generation}

Theoretical datasets for larvae, nymphs and adults were generated from the negative binomial distribution using the 'rnbinom' function in package 'base' within the $\mathrm{R}$ statistical software environment, available from www.r-project.org. Three parameters were required to simulate the data; the 'number of observations', $n$, (the host population size, set at 500 for all simulations), 'size' (also known as the dispersion parameter or the $k$-exponent of the negative binomial distribution, hereafter referred to as the ' $k$-exponent' or ' $k$ ') and ' $m u$ ' (the mean, in this case the mean number of each tick life stage present on the small mammal population). For each life stage, datasets were created assuming 3 distributions using a range of values of the $k$-exponent. The first dataset had a starting value of $k=0 \cdot 1$ and increased in increments of $0 \cdot 1$ until $k=1 \cdot 0$, thus representing natural levels of aggregation of ticks on small mammals in Europe (Randolph et al. 1999). The 
second dataset represented a dispersed independent distribution of ticks on hosts beginning at $k=1.5$ and increasing in increments of 0.5 until $k=30.5$ at which point the variance was approximately equal to the mean and the negative binomial distribution became Poisson distributed $($ mean $=6$, variance $=6 \cdot 67$, goodness of fit to the Poisson distribution $\chi^{2}=18 \cdot 5$, D.F. $=14, P=0 \cdot 184)$. The third dataset represented a coincident aggregated distribution and utilized data generated for the independent aggregation of ticks in dataset $1(0 \cdot 1 \leqslant k \leqslant 1 \cdot 0)$, but in this case $80 \%$ of nymphs that were previously independently distributed among the host population were redistributed randomly among the $20 \%$ of individuals most parasitized by larval ticks, thus conforming to the $80 / 20$ rule and achieving a coincident aggregated distribution with values of $k$ ranging between $0 \cdot 1$ and $1 \cdot 0$. As adult ticks were so rare among the datasets $(m u=0 \cdot 001)$, all adults were re-distributed randomly among the top $20 \%$ of individuals most parasitized by larval ticks for the coincident aggregated dataset. Therefore, 86 individual datasets were created each for larval, nymphal and adult ticks, 10 for the independent aggregated distribution $(0 \cdot 1 \leqslant k \leqslant 1 \cdot 0)$, 66 for dispersed distribution $(1 \cdot 5 \leqslant k \leqslant 30 \cdot 5)$ and 10 for the coincident aggregated distribution $(0 \cdot 1 \leqslant k \leqslant 1 \cdot 0)$. Increments of $0 \cdot 1$ were used from $0 \cdot 1$ to $1 \cdot 0$ to increase the sample size for this range of values of the $k$-exponent, as they represented natural levels of aggregation as previously stated.

Values of ' $m u$ ', the average number of each tick life stage were set as $6,0 \cdot 2$ and 0.001 for larvae, nymphs and adults respectively (cited by Hartemink et al. 2008). Although the presence of adult ticks is rare among small mammals the model is parameterized to indicate whether the pathogen is likely to become established or persist in small mammal cycles and we therefore assume that the tick population is supported by larger mammals within the system e.g. sheep, hares or deer. The data generated for larvae, nymphs and adults were used to calculate parameters within the $R_{0}$ model of Hartemink et al. (2008) that are affected by the degree of aggregation of ticks on hosts i.e. those involved with co-feeding: $C_{\mathrm{LL}}, C_{\mathrm{NL}}, C_{\mathrm{AL}}$, $C_{\mathrm{LN}}, C_{\mathrm{NN}}, C_{\mathrm{AN}}, C_{\mathrm{LA}}, C_{\mathrm{NA}}, C_{\mathrm{AA}}$ (for example, $C_{\mathrm{LL}}$ is 'the mean number of larvae co-feeding with a larva', see Table 1 for a full list of parameter descriptions). A set of these parameters was produced for each of the 86 individual datasets across the range of the dispersed distribution $(1 \cdot 5 \leqslant k \leqslant 30 \cdot 5)$, the independent aggregated distribution $(0 \cdot 1 \leqslant k \leqslant 1 \cdot 0)$ and the coincident aggregated distribution $(0 \cdot 1 \leqslant k \leqslant 1)$.

\section{The next generation matrix}

When diseases are transmitted directly, estimating $R_{0}$ is straightforward but vector-borne diseases are more complex as they utilize different 'infected types' i.e. hosts and vectors. This is made increasingly complex
Table 1. Ecological parameters for Ixodes ricinus

(Adapted from Hartemink et al. 2008.)

\begin{tabular}{|c|c|c|}
\hline Parameter & Description & Estimate $\uparrow$ \\
\hline$E$ & Eggs per adult & $2000^{1,2}$ \\
\hline$s_{\mathrm{L}}$ & $\begin{array}{l}\text { Survival probability from } \\
\text { egg to feeding larvae }\end{array}$ & $0 \cdot 05^{1}$ \\
\hline$s_{\mathrm{N}}$ & $\begin{array}{l}\text { Survival probability } \\
\text { from feeding larvae to } \\
\text { feeding nymph }\end{array}$ & $0 \cdot 1^{1}$ \\
\hline$s_{\mathrm{A}}$ & $\begin{array}{l}\text { Survival probability } \\
\text { from feeding nymph to } \\
\text { feeding adult }\end{array}$ & $0 \cdot 1^{1}$ \\
\hline$C_{\mathrm{LL}}$ & $\begin{array}{l}\text { Mean number of larvae } \\
\text { co-feeding with a larva }\end{array}$ & $6-20^{*}$ \\
\hline$C_{\mathrm{NL}}$ & $\begin{array}{l}\text { Mean number of nymphs } \\
\text { co-feeding with a larva }\end{array}$ & $0 \cdot 1-0 \cdot 2^{*}$ \\
\hline$C_{\mathrm{AL}}$ & $\begin{array}{l}\text { Mean number of adults } \\
\text { co-feeding with a larva }\end{array}$ & $0^{*}$ \\
\hline$C_{\mathrm{LN}}$ & $\begin{array}{l}\text { Mean number of larvae } \\
\text { co-feeding with a nymph }\end{array}$ & $4 \cdot 2-7 \cdot 5^{*}$ \\
\hline$C_{\mathrm{NN}}$ & $\begin{array}{l}\text { Mean number of nymphs } \\
\text { co-feeding with a nymph }\end{array}$ & $2-3 \cdot 7^{*}$ \\
\hline$C_{\mathrm{AN}}$ & $\begin{array}{l}\text { Mean number of adults } \\
\text { co-feeding with a nymph }\end{array}$ & $0-0 \cdot 01^{*}$ \\
\hline$C_{\mathrm{LA}}$ & $\begin{array}{l}\text { Mean number of larvae } \\
\text { co-feeding with an adult }\end{array}$ & $0-22^{*}$ \\
\hline$C_{\mathrm{NA}}$ & $\begin{array}{l}\text { Mean number of nymphs } \\
\text { co-feeding with an adult }\end{array}$ & $0^{*}$ \\
\hline$C_{\mathrm{AA}}$ & $\begin{array}{l}\text { Mean number of adults } \\
\text { co-feeding with an adult }\end{array}$ & $0^{*}$ \\
\hline$N_{\mathrm{LH}}$ & $\begin{array}{l}\text { Average number larvae } \\
\text { on competent host }\end{array}$ & $6^{3,4,5}$ \\
\hline$N_{\mathrm{NH}}$ & $\begin{array}{l}\text { Average number nymphs } \\
\text { on competent host }\end{array}$ & $0 \cdot 2^{4,5}$ \\
\hline$N_{\mathrm{AH}}$ & $\begin{array}{l}\text { Average number adults } \\
\text { on competent host }\end{array}$ & $0 \cdot 001^{6}$ \\
\hline$D_{\mathrm{L}}$ & Days of attachment of larva & $2 \cdot 5^{4}$ \\
\hline$D_{\mathrm{N}}$ & Days of attachment of nymph & $3 \cdot 5^{6}$ \\
\hline$D_{\mathrm{A}}$ & Days of attachment of adult & $12^{6}$ \\
\hline
\end{tabular}

$\uparrow$ Numbers in superscript refer to the following sources cited by Hartemink et al. (2008): ${ }^{1}$ Randolph and Craine (1995); ${ }^{2}$ Randolph (2004); ${ }^{3}$ Randolph et al. (1999); ${ }^{4}$ Gray (2002): ${ }^{5}$ Humair et al. (1999): ${ }^{6}$ S.E. Randolph, unpublished manuscript. *Parameters calculated in the current study across a range of values of the $k$-exponent. Please refer to the Supplementary data (online version only) for equations used to calculate each element within the next generation matrix and for the structure of the matrix.

when additional transmission routes and host and vector types are present; however, the nextgeneration matrix provides a method to incorporate these complexities. The matrix is based on the concept of types at birth i.e. the different states at which individuals become infected. Hartemink et al. (2008) identified 5 types at birth for tick-borne infections, (1) eggs (via transovarial transmission), (2) larvae, (3) nymphs, and (4) adults (via bloodmeals) and (5) vertebrate hosts. For a system with 5 types at birth the next generation matrix will be a $5 \times 5$ matrix of elements with every type at birth being infected by another (see Supplementary material for elements and the structure of the next generation matrix, online 
Table 2. Ecological parameters for Borrelia burgdorferi s.l. and tick-borne encephalitis virus

(Taken from Hartemink et al. 2008.)

\begin{tabular}{llll}
\hline \hline Parameter & Description & B. burgdorferi s.l. & TBE virus $\dagger$ \\
\hline$i$ & Systemic infection duration & 120 days $^{1}$ & 2 days $^{1}$ \\
$\theta$ & Efficiency from tick to tick & $0 \cdot 56^{2}$ & $0 \cdot 55^{3}, 4$ \\
$p_{\mathrm{L}}$ & Efficiency from competent host to larva & $0 \cdot 5^{5}$ & $0 \cdot 8^{6}$ \\
$p_{\mathrm{N}}$ & Efficiency from competent host to nymph & $0 \cdot 5^{6}$ & $0 \cdot 8^{6}$ \\
$p_{\mathrm{A}}$ & Efficiency from competent host to adult & $0 \cdot 4^{7}$ & $0 \cdot 8^{6}$ \\
$q_{\mathrm{L}}$ & Efficiency from larva to competent host & $0 \cdot 8^{6}$ & $0 \cdot 9^{6}$ \\
$q_{\mathrm{N}}$ & Efficiency from nymph to competent host & $0 \cdot 8^{6}$ & $0 \cdot 9^{6}$ \\
$q_{\mathrm{A}}$ & Efficiency from adult to competent host & $0 \cdot 8^{6}$ & $0 \cdot 9^{6}$ \\
$r_{\mathrm{A}}$ & Efficiency from adult to egg & $0 \cdot 1^{8}$ & $0 \cdot 001^{9}$ \\
\hline \hline
\end{tabular}

+ Numbers in superscript refer to the following sources cited by Hartemink et al. (2008): ${ }^{1}$ Randolph et al. (1996); ${ }^{2}$ Gern and Rais (1996); ${ }^{3}$ Labuda et al. (1993); ${ }^{4}$ Labuda et al. (1997); ${ }^{5}$ Randolph and Craine (1995); ${ }^{6}$ S.E. Randolph, unpublished manuscript; ${ }^{7}$ Kurtenbach et al. (1994); ${ }^{8}$ Hubálek and Halouzka (1998). Please refer to the Supplementary data (online version only) for equations used to calculate each element within the next generation matrix and for the structure of the matrix.

version only). If we consider the element $k_{31}$ : the number of nymphs (type at birth 3 ) infected by a tick infected as an egg (type at birth 1$)$, which has the formula $k_{31}=\left(s_{\mathrm{L}} \boldsymbol{\sigma}_{\mathrm{LN}} \mathrm{C}_{\mathrm{NL}}+s_{\mathrm{L}} s_{\mathrm{N}} \emptyset_{\mathrm{NN}} \mathrm{C}_{\mathrm{NN}}+s_{\mathrm{L}} s_{\mathrm{N}}\right.$ $\left.s_{\mathrm{A}} \sigma_{\mathrm{AN}} \mathrm{C}_{\mathrm{NA}}\right) h_{c}$, it has 3 terms as ticks infected as eggs can infect nymphs after their first, second and third bloodmeals while the survival to each stage $(s)$, the transmission efficiency from tick to tick $(ø)$ and the aggregation of ticks (C) is taken into account. Of course, some of the elements of the matrix are not possible and therefore equal 0 , for example, $k_{15}$, as tick eggs cannot be infected by a vertebrate host.

\section{Calculation of $R_{0}$}

Values of $R_{0}$ were calculated for B. burgdorferi s.l. and tick-borne encephalitis virus (TBEV) using the next generation matrix of Hartemink et al. (2008). Tick-related parameters involved with co-feeding transmission derived from datasets described above $\left(C_{\mathrm{LL}}-C_{\mathrm{AA}}\right.$, Table 1$)$ and previously published pathogen-specific parameters (Tables 1 and 2) were entered into equations to calculate each element of the matrix (please see Supplementary material for equations used to calculate each element, online version only). Previously published parameters were held constant while each of the 86 parameter sets $\left(C_{\mathrm{LL}}-C_{\mathrm{AA}}\right)$ were substituted into the model in turn, thus allowing values of $R_{0}$ for each pathogen to be calculated as a function of the degree of aggregation. $R_{0}$ was calculated via the spectral decomposition of the parameterized next-generation matrix using the eigen(matrix) function in package 'base' in R. Values of $R_{0}$ were plotted as a function of the $k$-exponent and non-linear regression performed (Sigmaplot 10, Systat Software Inc.) for 2 datasets, one as the dispersed distribution approached the independent aggregated distribution and the second as dispersed distribution approached the coincident aggregated distribution.
To give an indication of the mean effect each degree of aggregation had on the basic reproduction number in relation to the proportion of transmissioncompetent hosts on which I. ricinus is feeding, values of $R_{0}$ were averaged across the datasets for each of the 3 levels of aggregation and the difference between the means of each distribution $\left(\Delta R_{0}\right)$ calculated for a range of values of $h_{c},(10-100 \%$ at $10 \%$ intervals). In the current study we define small mammals as 'competent hosts' as they facilitate both transstadial and co-feeding transmission for both pathogens and are considered to be the most important reservoirs of these pathogens in Europe. Although sheep support co-feeding transmission of $B$. burgdorferi s.l., they are not thought to be important for the epidemiology of this pathogen in most of its range (Hartemink et al. 2008).

\section{RESULTS}

Increasing levels of aggregation of ticks on hosts led to an increase in values of $R_{0}$ for both pathogens. When values of $R_{0}$ were plotted as a function of $k$ the effect of aggregation on $R_{0}$ values could be clearly seen. The independent aggregated distribution formed at values of the $k$-exponent between $0 \cdot 1$ and $1 \cdot 0$ caused values of $R_{0}$ to increase in comparison to values observed when ticks were dispersed, leading to an inverse first-order polynomial relationship between $k$ and $R_{0}$ when the dispersed distribution converged on the independent aggregated distribution (non-linear regression, $\mathrm{R}_{a d j}^{2}=0 \cdot 87, \quad \mathrm{~F}=478 \cdot 66$, D.F. $=68, P<0 \cdot 0001, f=3 \cdot 87+(0 \cdot 02 / x),(B$. burgdorferi s.1.) and $\mathrm{R}_{a d j}^{2}=0 \cdot 47, \mathrm{~F}=115 \cdot 94$, D.F. $=68, P<0 \cdot 0001$, $f=0 \cdot 73+(0 \cdot 001 / x)(\mathrm{TBEV})$, adjusted $\mathrm{R}^{2}, \mathrm{~F}$-value, degrees of freedom, $P$-value and relationship of the line of the first order polynomial, Fig. 1a and b). The coincident aggregated distribution also caused an increase in $R_{0}$ relative to the dispersed distribution but to a greater degree than the independent 
(a)

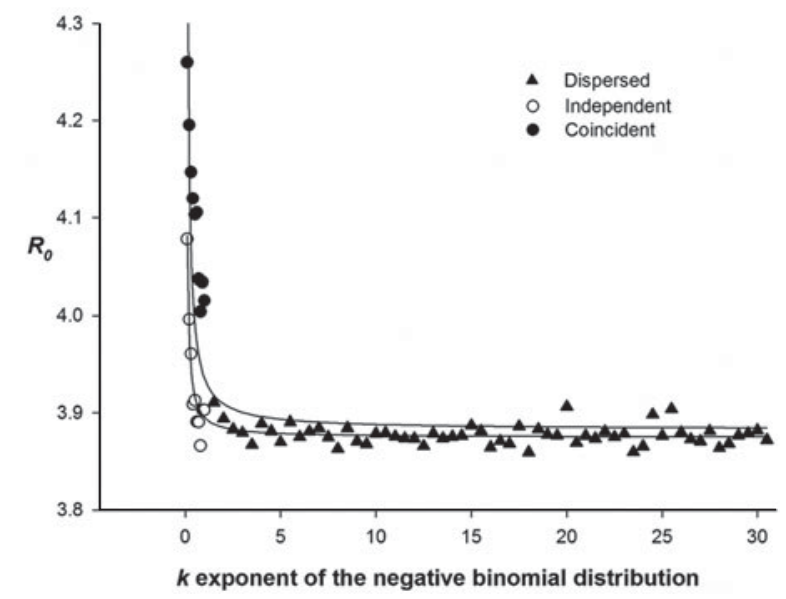

(b)

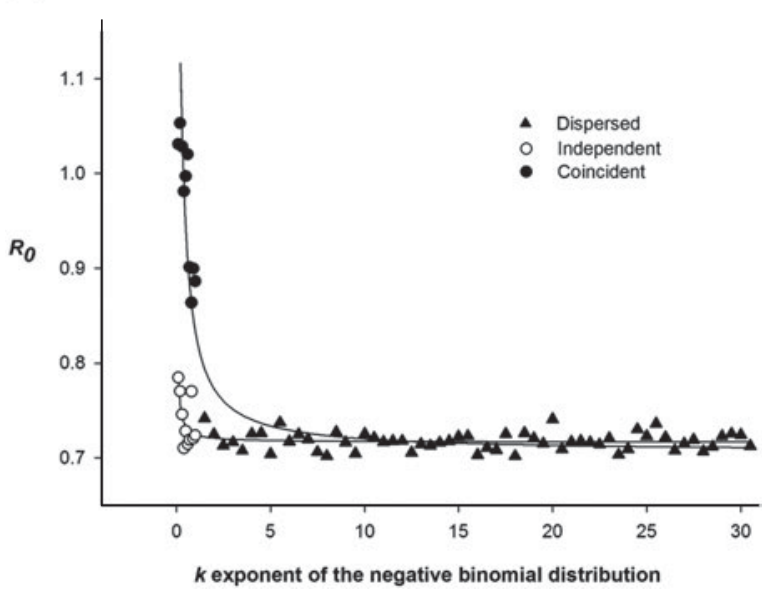

Fig. 1. Values of $R_{0}$ calculated for (a) Borrelia burgdorferi s.l. and (b) tick-borne encephalitis virus, plotted as a function of the degree of aggregation of ticks on small mammal hosts ( $k$-exponent of the negative binomial distribution) ranging from dispersed $(1 \cdot 5 \leqslant k \leqslant 30 \cdot 5)$ to independent aggregated $(0 \cdot 1 \leqslant k \leqslant 1)$ and from the dispersed $(1 \cdot 5 \leqslant k \leqslant 30 \cdot 5)$ to the coincident aggregated distributions $(0 \cdot 1 \leqslant k \leqslant 1)$. Small values of the $k$-exponent indicate high levels of aggregation, larger values indicate low levels of aggregation. Plots of firstorder polynomial relationships between $k$ and $R_{0}$ for the dispersed distribution converging on the independent aggregation and for the dispersed distribution converging on the coincident aggregated distribution for each pathogen are also presented.

aggregated distribution. This relationship also followed an inverse first-order polynomial $\left(\mathbf{R}_{a d j}^{2}=0 \cdot 77\right.$, $\mathrm{F}=231 \cdot 66$, D.F. $=68, P<0 \cdot 0001, f=3 \cdot 88+(0 \cdot 05 / x)$, (B. burgdorferi s.1.) and $\mathrm{R}_{a d j}^{2}=0.62, \quad \mathrm{~F}=60 \cdot 74$, D.F. $=68, P<0 \cdot 0001, f=0 \cdot 72+(0 \cdot 001 / x)$ (TBEV).

Average values of $R_{0}$ were increased for both pathogens with increasing levels of aggregation relative to those obtained when ticks followed a dispersed distribution, these values also increased in relation $h_{c}$ (Table 3.). For B. burgdorferi s.l., the average value of $R_{0}$ increased by a minimum of 0.012 $(0.75 \%)$ from 1.594 to 1.606 at $h_{c}=10 \%$ and by a maximum of $0 \cdot 066(1 \cdot 49 \%)$ from 4.444 to 4.510 at $h_{c}=100 \%$ when ticks followed the independent aggregated distribution relative to the dispersed distribution. When ticks followed a coincident aggregated distribution the average value of $R_{0}$ increased by $0.033(2.07 \%)$ from 1.594 to 1.627 at $h_{c}=10 \%$ and by $0 \cdot 297(6 \cdot 68 \%)$ from $4 \cdot 444$ to 4.741 at $h_{c}=100 \%$ relative to the dispersed distribution. Therefore, the threshold value of $R_{0}>1$ was achieved for $B$. burgdorferi s.l. regardless of the level of aggregation of ticks on hosts and even when the proportion of competent hosts on which I. ricinus had fed was very low $(10 \%)$. In the case of TBEV, the average value of $R_{0}$ increased by $0.003(1.32 \%)$ from 0.227 to 0.230 at $h_{c}=10 \%$ and by $0.028(3.26 \%)$ from 0.858 to 0.886 at $h_{c}=100 \%$ when ticks followed an independent aggregated distribution relative to a dispersed distribution. When ticks followed a coincident aggregated distribution, the effect was much greater, the average $R_{0}$ value increased by 0.029 $(12.78 \%)$ from 0.227 to 0.256 at $h_{c}=10 \%$ and by 0.342 (39.86\%) from 0.858 to 1.200 at $h_{c}=100 \%$. In contrast to $B$. burgdorferi s.l., values of $R_{0}$ for TBEV only achieved the threshold value when $h_{c}$ reached $80 \%$ and only when ticks followed a coincident aggregated distribution. In this case the coincident aggregated distribution of ticks on hosts as well as ticks feeding on a high proportion of transmission competent hosts is essential for the threshold value of $R_{0}$ to be achieved and the pathogen to persist.

\section{DISCUSSION}

Using different levels of aggregation from the dispersed to the independent aggregated to the coincident aggregated we showed that values of $R_{0}$ became elevated with progressive levels of aggregation, making it increasingly likely for these pathogens to become established and to persist. In the case of Borrelia burgdorferi s.l., values of $R_{0}$ were always above the threshold value for the establishment of the disease and therefore the level of aggregation of ticks on hosts and the proportion of competent hosts on which ticks are feeding has little effect on whether this pathogen becomes established or not. In contrast, the threshold value of $R_{0}$ for TBEV was only achieved when the proportion of transmissioncompetent hosts on which ticks were feeding reached $80 \%$ and only when the ticks followed a coincident aggregated distribution. This suggests that any decrease in the proportion of competent hosts on which ticks are feeding, for example through vaccination or increasing the diversity of potential vertebrate hosts (assuming they are less competent transmission hosts), will cause a reduction in $R_{0}$ and the pathogen will become less likely to become established or to persist. For TBEV, the distribution of ticks on hosts has a much more definitive role in whether the pathogen becomes established or not. 


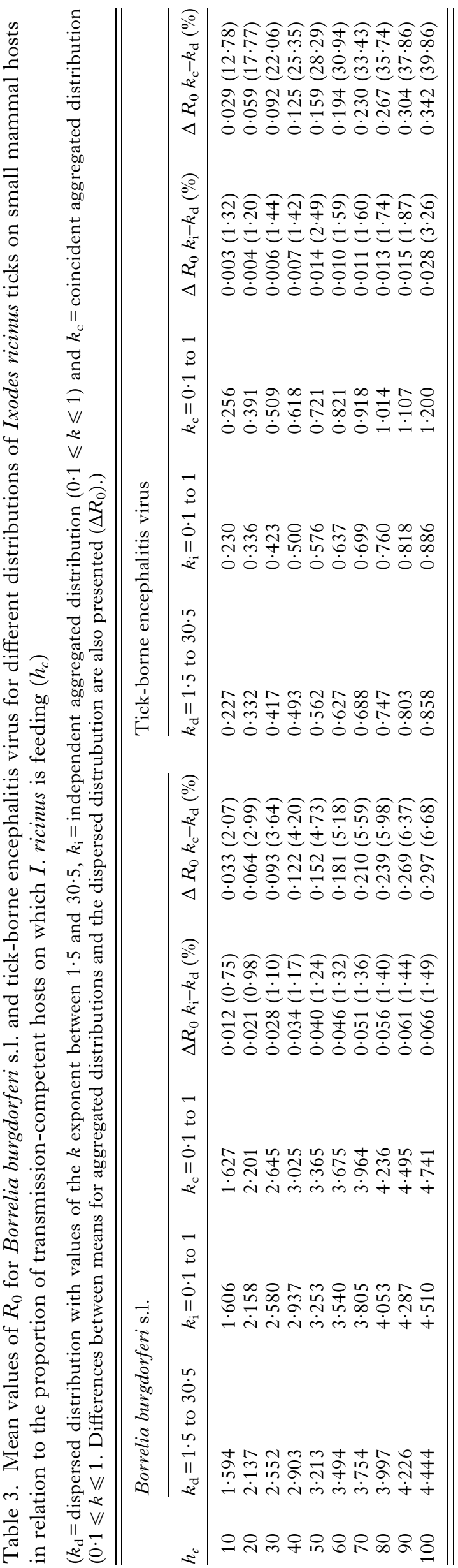

Without the increased transmission efficiency afforded to the pathogen via this enhanced potential for the co-feeding transmission route this pathogen would not become established in small mammal hosts at all. Results of the current study are consistent with those of Hartemink et al. (2008) and Randolph et al. (1996) who also found that the threshold value of $R_{0}$ was easily achieved for $B$. burgdorferi s.l. with or without co-feeding transmission but the threshold value for TBEV was only achieved when co-feeding transmission was present.

The differential response of these two pathogens to co-feeding transmission lies in differences in their systemic infection duration (Randolph et al. 1996). The systemic infection of B. burgdorferi s.l. in a rodent host is 120 days but only 2 days for TBEV (Randolph et al. 1996). When considering transstadial transmission, rodents infect ticks with B. burgdorferi s.l. over a long period of time, furnishing the system with large numbers of infected ticks that in turn infect large numbers of hosts. This leads to a greater overall level of transmission between hosts and vectors and allows values of $R_{0}$ to be maintained above the threshold value for establishment. In contrast, rodents can only infect ticks with TBEV over a short period. Thus many fewer ticks become infected with TBEV than with $B$. burgdorferi s.l. and values of $R_{0}$ for TBEV are reduced so that they do not reach the threshold value for establishment of the pathogen. However, the extra boost in overall transmission efficiency supplied by co-feeding transmission, driven by the coincident aggregated distribution of ticks, is enough in some cases to increase values of $R_{0}$ above the threshold value. This explains why natural cycles of $B$. burgdorferi s.l. are widespread while those of TBEV are highly focal and patchy in distribution; only certain locations provide the correct climatic condition for the seasonal synchrony of larvae and nymphs on small mammal hosts that contribute to the coincident aggregated distribution that drives co-feeding transmission (Randolph et al. 1996; Randolph and Rogers, 2000). Warm summers allow the rapid development of ticks while rapid autumn cooling sends larvae into winter diapause causing them to emerge in synchrony with nymphs in spring and facilitates the coincident aggregation of ticks on hosts and, therefore, cofeeding transmission (Randolph and Rogers, 2000; Gatewood et al. 2009). In contrast to TBEV, locations with climatic conditions that promote the activity of nymphs prior to larvae often have the strongest transmission cycles for B. burgdorferi s.l (Kurtenbach et al. 2006). As co-feeding transmission is less important for B. burgdorferi s.1, this activity profile promotes the persistence of this pathogen as it allows time for vertebrate hosts to become infected by nymphs, develop a systemic infection, and to subsequently infect larvae. In addition, genospecies of Borrelia that persist for longer in vertebrate hosts 
may be favoured by climate-driven asynchrony of immature ticks while those genospecies that have a shorter systemic may be driven by seasonal synchrony (Kurtenbach et al. 2006; Gatewood et al. 2009). This can lead to geographical variations in the distribution of Borrelia genospecies driven by climate (Gatewood et al. 2009). While the degree of aggregation of ticks on hosts at a given point in time has implications for pathogen transmission, there is also a temporal aspect due to the seasonality of immature ticks in response to climate.

While the coincident aggregated distribution caused an increase in $R_{0}$ so did the independent aggregated distribution, albeit to a lesser extent. In this case, it is not that individuals have an increased chance of hosting both larvae and nymphs but that the independent aggregation of larvae and nymphs is such that occasionally some individuals will host nymphs and, by chance, large numbers of larvae at the same time. This will lead to an 'accidental' increase in $R_{0}$ but to a lesser extent than when individuals consistently host nymphs and larvae together as under the coincident aggregated distribution.

In addition to the climatic conditions that allow synchronous feeding of larval and nymphal ticks, a number of other biotic factors are involved. Questing larvae are themselves clumped in their environment as they emerge from egg masses laid down by females (Randolph and Steele, 1985; Daniels and Fish, 1990). Sexually active adult male hosts often cover wider ranges than other individuals within the population and so may encounter more ticks in the environment (Randolph, 1977; Boyer et al. 2010). These individuals are often the largest within a population (due to maturity and sexual size dimorphism) and there is evidence to suggest that larger individuals have greater tick loads than smaller individuals (Gallivan and Horak, 1997; Harrison et al. 2010). Sexually active males also have higher levels of testosterone that may depress innate and acquired resistance to ticks in small mammal hosts and lead to increased attachment rates (Hughes and Randolph, 2001). As a result of these factors affecting the exposure and susceptibility of individuals to parasites, adult males are often the ' $20 \%$ ' in the ' $80 / 20$ rule' i.e. they are the fraction of the host population that contributes most to the transmission of parasites and vector-borne diseases (Perkins et al. 2003; Ferrari et al. 2004; Bouchard et al. 2011). Perkins et al. (2003) demonstrated that within a population of Apodemus flavicollis, $20 \%$ of the hosts were responsible for $74 \%$ to $94 \%$ of transmission of TBEV to ticks and that adult males of high body mass were the most likely members of this group. Similarly, Ferarri et al. (2004) found that the $20 \%$ of A. flavicollis most infected with the nematode, Heligmosomoides polygyrus, (consisting of $62 \%$ males) accounted for $73 \%$ of the eggs expelled in faeces.
Although the aggregation of ticks on certain individuals, such as adult males, is common there may be exceptions and this will have consequences for the persistence of tick-borne pathogens. If tick life stages aggregate on different hosts temporally, for example, nymphs on adult males early in the season as a result of elevated testosterone or increased movement, and larvae on pregnant females or juveniles later in the season due to reduced immunity, then pathogens such as B. burgdorferi s.l would be less likely to persist (see Mannelli et al. 2012).

Identifying the most important demographic for the transmission of pathogens within a population has important implications for their control. By targeting the fraction of the population most accountable for the transmission of parasites, transmission potential can be reduced. Conversely, targeting individuals in the population outside the important ' $20 \%$ ' of key hosts may have little or no effect on the overall epidemiology pathogens (Perkins et al. 2003; Ferrari et al. 2004). The development of vaccines for B. burgdorferi s.l. in natural rodent hosts, such as white-footed mice, Peromyscus leucopus, has focussed on outer surface protein A (Osp A) which is involved with the attachment of spirochetes in the midgut of ticks (Pal et al. 2000). Recent field studies have indicated that vaccination of small mammal reservoirs may be a viable option for controlling Lyme borreliosis. Tsao et al. (2004) demonstrated that the prevalence of $B$. burgdorferi s.l. infection in I. scapularis could be reduced by between $19 \%$ and $25 \%$ via vaccination of white-footed mice while Telford et al. (2011) suggested that over half the population of white-footed mice could be targeted with nest-box deployed bait that could be used for vaccination. Although these measures are likely to reduce the number of nymphs infected with B. burgdorferi s.l. in the environment and, therefore, the risk to public health, data from the current study suggest that they are unlikely to eliminate the pathogen from the system altogether.

The current study uses the most up-to-date and biologically relevant model of the basic reproduction number, $R_{0}$, for tick-borne pathogens to investigate how the degree of aggregation of ticks on small mammal hosts, from the dispersed to the independent aggregated to the coincident aggregated distribution, affects the persistence of tick-borne pathogens in enzootic small mammal cycles. In addition, the relationship between the $k$-exponent of the negative binomial distribution and $R_{0}$ is defined for the first time.

\section{ACKNOWLEDGEMENTS}

This work was supported by a University of Pretoria Postdoctoral Fellowship awarded to A. Harrison and the DSTNRF South African Research Chair of Behavioural Ecology and Physiology awarded to N.C. Bennett. 


\section{REFERENCES}

Anderson, R. M. and May, R. M. (1990). Modern vaccines: Immunisation and herd immunity. Lancet 335, 641-645.

Bouchard, C., Beauchamp, G., Trudel, L., Milford, F., Lindsay, L. R., Belanger, D. and Ogden, N.H. (2011). Associations between Ixodes scapularis ticks and small mammal hosts in a newly endemic zone in southeastern Canada: Implications for Borrelia burgdorferi transmission. Ticks and Tick-borne Diseases 2, 183-190.

Boyer, N., Réale, D., Marmet, J., Pisanu, B. and Chapuis, J. L. (2010). Personality, space use and tick load in an introduced population of Siberian chipmunks Tamias sibiricus. Fournal of Animal Ecology 79, 538-547.

Caraco, T., Glavankov, S., Chen, G., Flaherty, J. E., Ohsumi, T. K. and Szymanksi, B. K. (2002). Stage-structured infection transmission and a spatial epidemic: a model for Lyme disease. American Naturalist $\mathbf{1 6 0}$ 348-359.

Crofton, H. (1971). A quantitative approach to parasitism. Parasitology 62 179-193.

Daniels, T. J. and Fish, D. (1990). Spatial distribution and dispersal of unfed larval Ixodes dammini (Acari: Ixodidae) in southern New York. Environmental Entomology 19,1029-1033.

Diekmann, O., Heesterbeek, J. A. P. and Metz, J. A. J. (1990). On the definition and the computation of the basic reproduction ratio $\mathrm{R}_{0}$ in models for infectious diseases in heterogeneous populations. Fournal of Mathematical Biology 28, 365-382.

Dobson, A. P. and Merenlender, A. (1991). Coevolution of macroparasites and their hosts. In Parasite-host Associations: Coexistence or Conflict? (ed. Toft, C. A., Aeschlimann, A. and Bolis, L.), pp. 83-101. Oxford University Press, Oxford, UK.

Ferrari, N., Cattadori, I. M., Nespereira, J., Rizzoli, A. and Hudson, P. J. (2004). The role of host sex in parasite dynamics: field experiments on the yellow-necked mouse Apodemus flavicollis. Ecology Letters 7, 88-94.

Gallivan, G. and Horak, I. (1997). Body size and habitat as determinants of tick infestations of wild ungulates in South Africa. South African fournal of Wildlife Research 27, 63-70.

Gatewood, A. G., Liebman, K. A., Vourc'h, G., Bunikis, J., Hamer, S. A., Cortinas, R., Melton, F., Cislo, P., Kitron, U. and Tsao, J. (2009). Climate and tick seasonality are predictors of Borrelia burgdorferi genotype distribution. Applied and Environmental Microbiology 75, 2476-2483

Gern, L. and Rais, O. (1996). Efficient transmission of Borrelia burgdorfer between cofeeding Ixodes ricinus ticks (Acari: Ixodidae). Fournal of Medical Entomology 33, 189-192.

Ghosh, M. and Pugliese, A. (2004). Seasonal population dynamics of ticks, and its influence on infection transmission: a semi-discrete approach. Bulletin of Mathematical Biology 66, 1659-1684.

Goodman, J. L., Dennis, D. T. and Sonenshine, D. E. (2005). Tick-borne diseases of humans. 1st Edn. American Society for Microbiology, Washington.

Gray, J. S. (2002). Biology of Ixodes species ticks in relation to tick-borne zoonoses. Wiener klinische Wochenschrift 114, 473-478.

Harrison, A., Montgomery, W. I. and Bown, K. J. (2011). Investigating the persistence of tick-borne pathogens via the $\mathrm{R}_{0}$ model. Parasitology 138 896-905.

Harrison, A., Scantlebury, M. and Montgomery, W. I. (2010). Body mass and sex-biased parasitism in wood mice Apodemus sylvaticus. Oikos 9999.

Hartemink, N. A., Randolph, S. E., Davis, S. A. and Heesterbeek, J. A. P. (2008). The basic reproduction number for complex disease systems: Defining $\mathrm{R}_{0}$ for tick-borne infections. The American Naturalist 171, 743-754.

Hubálek, Z. and Halouzka, J. (1998). Prevalence rates of Borrelia burgdorferi sensu lato in host-seeking Ixodes ricinus ticks in Europe. Parasitology Research 84, 167-172.

Hudson, P. J., Norman, R., Laurenson, M. K., Newborn, D. Gaunt, M., Jones, L., Reid, H., Gould, E., Bowers, R., and Dobson, A. (1995). Persistence and transmission of tick-borne viruses: Ixodes ricinus and louping-ill virus in red grouse populations. Parasitology 111, S49-S58.

Hughes, V. L. and Randolph, S. E. (2001). Testosterone depresses innate and acquired resistance to ticks in natural rodent hosts: a force for aggregated distributions of parasites. Fournal of Parasitology 87, 49-54.

Humair, P.F., Rais, O. and Gern, L. (1999). Transmission of Borrelia afzelii from Apodemus mice and Clethrionomys voles to Ixodes ricinus ticks: differential transmission pattern and overwintering maintenance. Parasitology 118, 33-42.
Jongejan, F. and Uilenberg, G. (2004). The global importance of ticks. Parasitology 129, 3-14.

Koch, R. (1999). 80/20 Principle: The Secret to Success by Achieving More with Less. 1st Edn. Currency, New York, USA.

Kurtenbach, K., Hanincová, K., Tsao, J. I., Margos, G., Fish, D. and Ogden, N. H. (2006). Fundamental processes in the evolutionary ecology of Lyme borreliosis. Nature Reviews Microbiology 4, 660-669.

Kurtenbach, K., Dizij, A., Seitz, H. M., Margos, G., Moter, S. E. Kramer, M. D., Wallich, R., Schaible, U. E. and Simon, M. M. (1994) Differential immune responses to Borrelia burgdorferi in European wild rodent species influence spirochete transmission to Ixodes ricinus L. (Acari: Ixodidae). Infection and Immunity 62, 5344-5352.

Labuda, M., Jones, L.D., Williams, T., Danielova, V. and Nuttall, P. A. (1993). Efficient transmission of tick-borne encephalitis virus between cofeeding ticks. Fournal of Medical Entomology 30, 295-299.

Labuda, M., Kozuch, O. and Lys, J. (1997). Tickborne encephalitis virus natural foci in Slovakia: ticks, rodents, and... goats. In 4th International Potsdam Symposium on Tick-borne Diseases: Tick-borne Encephalitis and Lyme borreliosis (ed. Suss, J. and Kahl, O.), pp. 21-22. Pabst Science Publishers, Lengerich, Germany.

Mannelli, A., Bertolotti, L., Gern, L. and Gray, J. (2012). Ecology of Borrelia burgdorferi sensu lato in Europe: transmission dynamics in multihost systems, influence of molecular processes and effects of climate change. FEMS Microbiology Reviews (in the Press).

Norman, R., Bowers, R. G., Begon, M. and Hudson, P. J. (1999) Persistence of tick-borne virus in the presence of multiple host species: tick reservoirs and parasite mediated competition. Fournal of Theoretical Biology 200, 111-118.

Norman, R., Ross, D., Laurenson, M. K., and Hudson, P. J. (2004). The role of non-viraemic transmission on the persistence and dynamics of a tick borne virus-Louping ill in red grouse (Lagopus Lagopus scoticus) and mountain hares (Lepus timidus). Fournal of Mathematical Biology 48 119-134.

Ostfeld, R. S. and Keesing, F. (2000). Biodiversity and disease risk: the case of Lyme disease. Conservation Biology 14, 722-728.

Pal, U., De Silva, A. M., Montgomery, R. R., Fish, D., Anguita, J., Anderson, J. F., Lobet, Y. and Fikrig, E. (2000). Attachment of Borrelia burgdorferi within Ixodes scapularis mediated by outer surface protein A Fournal of Clinical Investigation 106, 561-569.

Perkins, S. E., Cattadori, I. M., Tagliapietra, V., Rizzoli, A.P. and Hudson, P. J. (2003). Empirical evidence for key hosts in persistence of a tick-borne disease. International fournal for Parasitology 33, 909-917.

Randolph, S. E. (1977). Changing spatial relationships in a population of Apodemus sylvaticus with the onset of breeding. The Fournal of Animal Ecology 46, 653-676.

Randolph, S. (1998). Ticks are not insects: consequences of contrasting vector biology for transmission potential. Parasitology Today 14, 186-192. Randolph, S. E. (2004). Tick ecology: processes and patterns behind the epidemiological risk posed by ixodid ticks as vectors. Parasitology 129 , 37-65.

Randolph, S.E. and Craine, N.G. (1995). General framework for comparative quantitative studies on transmission of tick-borne diseases using Lyme borreliosis in Europe as an example. Fournal of Medical Entomology 32, 765-777.

Randolph, S. E., Gern, L. and Nuttall, P. A. (1996). Co-feeding ticks: epidemiological significance for tick-borne pathogen transmission. Parasitology Today 12, 472-479.

Randolph, S. E., Miklisova, D., Lysy, J., Rogers, D. J. and Labuda, M. (1999). Incidence from coincidence: patterns of tick infestations on rodents facilitate transmission of tick-borne encephalitis virus. Parasitology $\mathbf{1 1 8}$ 177-186.

Randolph, S. E. and Rogers, D. J. (2000). Fragile transmission cycles of tick-borne encephalitis virus may be disrupted by predicted climate change. Proceedings of the Royal Society of London, B 267, 1741-1744.

Randolph, S. E. and Steele, G. M. (1985). An experimental evaluation of conventional control measures against the sheep tick, Ixodes ricinus (L.) (Acari: Ixodidae). II. The dynamics of the tick-host interaction. Bulletin of Entomological Research 75, 501-518.

Rosá, R. and Pugliese, R. (2007). Effects of tick population dynamics and host densities on the persistence of tick-borne infections. Mathematical Biosciences 208, 216-240.

Rosá, R., Pugliese, R., Norman, R. and Hudson, P. J. (2003). Thresholds for disease persistence in models for tick-borne infections including non-viraemic transmission, extended feeding and tick aggregation. Fournal of Theoretical Biology 224, 359-376.

Schmidt, K. A. and Ostfeld, R.S. (2001) Biodiversity and the dilution effect in disease ecology. Ecology 82, 609-619. 
Shaw, D. and Dobson, A. (1995). Patterns of macroparasite abundance and aggregation in wildlife populations: a quantitative review. Parasitology 111, 111-133

Telford III, S. R., Cunningham, J. A., Waltari, E. and Hu, L. (2011). Nest box-deployed bait for delivering oral vaccines to white-footed mice. Ticks and Tick-borne Diseases 2, 151-155.

Tsao, J. I., Wootton, J. T., Bunikis, J., Luna, M. G., Fish, D. and Barbour, A. G. (2004). An ecological approach to preventing human infection: vaccinating wild mouse reservoirs intervenes in the Lyme disease cycle. Proceedings of the National Academy of Sciences, USA 101, 1815918164.
Wilson, K., Bjørnstad, O., Dobson, A., Merler, S., Poglayen, G., Randolph, S., Read, A., Skorping, A., Hudson, P. and Rizzoli, A. (2002). Heterogeneities in macroparasite infections: patterns and processes. In The Ecology of Wildlife Diseases (ed. Hudson P. J., Rizzoli, A., Grenfell, B. T., Heesterbeek, H. and Dobson, A. P.), pp. 6-44. Oxford University Press, Oxford, UK.

Woolhouse, M. E. J., Dye, C., Etard, J. F., Smith, T., Charlwood, J., Garnett, G., Hagan, P., Hii, J., Ndhlovu, P. and Quinnell, R. (1997) Heterogeneities in the transmission of infectious agents: implications for the design of control programs. Proceedings of the National Academy of Sciences, USA 94, 338-342. 